Landespflegekammer RLP

\section{Krankenhauskeime wirksam bekämpfen}

— Pflegende sind die Berufsgruppe mit dem häufigsten Patientenkontakt. Um Hygienebestimmungen professionell umzusetzen, müssen die Rahmenbedingungen stimmen, fordert die Landespflegekammer Rheinland-Pfalz.

Die Studie europäischer Forscher über das Auftreten von Krankheitserregern in Krankenhäusern sorgte kürzlich für Aufmerksamkeit: Den Ergebnissen zufolge infizieren sich etwa 2,6 Millionen Patienten europaweit mit sogenannten Krankenhauskeimen. Die Daten wurden in den Jahren 2011 und 2012 in 30 Ländern erhoben. In Deutschland sollen es gemäß Schätzungen des Nationalen Referenzzentrums zur Überwachung von Krankenhausinfektionen an der Berliner Charité etwa 500.000 Infektionen geben. „Der häufigste Übertragungsweg sind die Hände. Daher ist für die beruflich Pflegenden die Handhygiene ein so eminent wichtiges Thema", betont Dr. Markus Mai, Präsident der Landespflegekammer Rheinland-Pfalz. „Die korrekte
Ausübung einer professionellen Handhygiene benötigt Zeit. Jede Reduzierung von Pflegenden kostet dabei Zeit und nimmt auch die Möglichkeit zur notwendigen Hygiene", warnt Mai vor den Folgen des Sparens beim Pflegepersonal. Es sei von besonderer Relevanz, dass gerade diese Berufsgruppe ausreichend Möglichkeiten zur Handhygiene erhalte. Erschwert werde diese auch in manchen Einrichtungen durch die bauliche Infrastruktur. An dieser Stelle gilt es aus Sicht des Kammerpräsidenten, nachzusteuern.

„Wer Personalbestände in der Pflege abbaut, um Kosten zu sparen, muss wissen, welch gefährliches Spiel er betreibt. Fehlt diese Zeit aufgrund nicht ausreichend besetzter Pflegestellen werden Infektionen billigend in Kauf genommen", konstatiert Mai. Die Forderung nach einer gesetzlich verankerten Personalbemessung in der Pflege sei daher zwingend notwendig.

www.pflegekammer-rlp.de
Zukunftsvision

\section{Landespflegekammer bald auch in NRW?}

— Nach Ansicht der CDU-Landtagsfraktion in NRW braucht nach Rheinland-Pfalz, Schleswig-Holstein und Niedersachsen nun auch NRW eine Pflegekammer. Ein entsprechender Antrag ist von den Christdemokraten an die Landesregierung gerichtet worden. Am 26. Oktober 2016 fand nun vor dem Ausschuss für Arbeit, Gesundheit und Soziales des Landtages von NRW eine Anhörung zur Pflegekammer statt. In seiner Stellungnahme fordert der DBfK Nordwest die Mitglieder des Ausschusses auf, sich für ein entsprechendes Gesetzgebungsverfahren stark zu machen. „Aufgrund der enormen Bedeutung von Pflegenden und der aktuell indiskutablen Arbeitsbedingungen erwarten wir einen von allen Parteien getragenen Willen zur Emanzipation der Pflegenden und zur Verbesserung der Arbeitsbedingungen für Pflegende", sagt Martin Dichter, Vorsitzender des DBfK Nordwest.

www.dbfk.de

\title{
Neuauflage
}

\section{Alles was Recht ist in Pflegeausbildung und Pflegeleitung}

_ Pflegeleistungen müssen hohen Qualitätsansprüchen genügen und patientengerecht erbracht werden. In dem Buch "Recht in der Pflege" von dem Fachanwalt für Arbeitsrecht, Prof. Thomas Weiß, werden auf der Grundlage wissenschaftlicher Erkenntnisse und praktischer Erfahrungen die rechtlichen Anforderungen an Personen

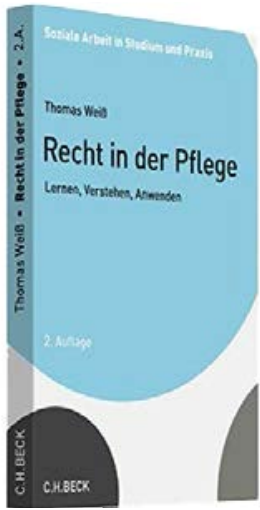
und Institutionen im Pflegebereich dargestellt und konkrete Ansätze für die Realisierung/Umsetzung durch Leitungskräfte in der Pflege aufgezeigt.

Zahlreiche Beispiele, Musterfälle und
Schaubilder sorgen für ein schnelles Verständnis der rechtlichen Zusammenhänge. Der Autor richtet sich in seinem Buch an Pflegekräfte, an Pflegende die sich für Leitungsaufgaben qualifizieren oder in solchen Tätigkeitsbereichen fundiert Entscheidungen treffen wollen bzw. müssen und an diejenigen, die die rechtlichen Grundlagen der Pflege lehren und vermitteln. Die aktualisierte Auflage des bewährten Lehrbuchs ist auf dem Stand von August 2016 und berücksichtigt alle Neuerungen im Bereich Gesetzgebung in der Pflege.

Vorschau! Die PflegeKollegs im Monat Januar:

Pflege im Team

Dienstplan - Führung - Lebensphasengerechtes Arbeiten

Pflege im Recht

Thomas Weiß. Recht in der Pflege. Lernen, Verstehen, Anwenden. C.H. Beck oHG 2016. ISBN 9783406665172; $€ 29,80$ (www.beck.de) 